\title{
Continuous wave terahertz radiation from an InAs/GaAs quantum-dot photomixer device
}

\author{
T. Kruczek, ${ }^{1}$ R. Leyman, ${ }^{1}$ D. Carnegie, ${ }^{1}$ N. Bazieva, ${ }^{1}$ G. Erbert, ${ }^{2}$ S. Schulz, ${ }^{3}$ C. Reardon, ${ }^{3}$ \\ S. Reynolds, ${ }^{1}$ and E. U. Rafailov ${ }^{1}$ \\ ${ }^{1}$ Photonics and Nanoscience Group, School of Engineering, Physics and Mathematics, University of Dundee, \\ Dundee DDI 4HN, United Kingdom \\ ${ }^{2}$ Ferdinand-Braun-Institute, Albert-Einstein-Straße 11, D-12489 Berlin, Germany \\ ${ }^{3}$ School of Physics and Astronomy, University of St. Andrews, St. Andrews, Fife KY16 9SS, United Kingdom
}

(Received 11 April 2012; accepted 9 August 2012; published online 23 August 2012; corrected 8 October 2012)

\begin{abstract}
Generation of continuous wave radiation at terahertz $(\mathrm{THz})$ frequencies from a heterodyne source based on quantum-dot (QD) semiconductor materials is reported. The source comprises an active region characterised by multiple alternating photoconductive and QD carrier trapping layers and is pumped by two infrared optical signals with slightly offset wavelengths, allowing photoconductive device switching at the signals’ difference frequency $\sim 1$ THz. @ 2012 American Institute of Physics. [http://dx.doi.org/10.1063/1.4747724]
\end{abstract}

Continuous wave $(\mathrm{CW})$ terahertz $(\mathrm{THz})$ optoelectronic devices and materials have seen considerable interest and development since the first conclusive demonstration by Brown et al. of the THz photomixer, the results of which were published in $1993,{ }^{1}$ and there is now a variety of effective solutions to the challenge of generating electromagnetic fields within the so-called "THz gap" between 0.1-10 THz. ${ }^{2}$ Early, THz optoelectronics were based on photoconductive (PC) switches and antennas $^{3}$ and optimised electro-optic materials such as ZnTe, ${ }^{4}$ typically driven by femtosecond pulsed Ti:Sapphire lasers, but these pulse-driven methods exhibit poor frequency tunability and low electro-optic efficiency and are inherently bulky, complex, expensive, and mechanically unstable.

$\mathrm{CW} \mathrm{THz}$ signals may be generated using semiconductor materials in which (photo) carrier lifetimes are limited to $1 \mathrm{ps}$ or shorter. Photoconductive material(s) may be optically pumped by a signal composed of two distinct, narrow linewidth modes with a few nm wavelength difference. This pump signal may be set up by the spatial combination of two coherent optical beams, or by using a single beam generated by a coherent multi-mode source. ${ }^{5} \mathrm{~A} \mathrm{THz}$ signal is the resultant difference frequency, the PC material is modulated at this frequency, and electron-hole pairs are generated and accelerated by the electric field set up by an integrated metallic antenna. As the material is electrically switched, a dc potential applied across the photoconductive antenna (PCA) is coupled through the device substrate and into free space, analogously to a traditional Hertzian-type dipole antenna.

The active PC material has been the most heavily researched aspect of $\mathrm{CW} \mathrm{THz} \mathrm{optoelectronics,} \mathrm{since} \mathrm{the} \mathrm{semi-}$ nal work into ultrafast semiconductors such as radiationdamaged silicon-on-sapphire ${ }^{6}$ and low-temperature-grown gallium arsenide (LT-GaAs). ${ }^{7,8}$ Photocarrier lifetimes here are limited by controllably introducing lattice deformations either during epitaxial growth or with post-process ion bombardment. However, this also means that several compromises are made in the materials' performance such as low carrier mobilities and poor thermal conductivity. The solution to these ultrafast PC material compromises may be quantum-dot
(QD's). QD's may be deposited during molecular beam epitaxy (MBE) as monolayers of self-assembled islands of III-V materials, driven by surface chemistry. They exhibit many curious optoelectronic properties, which are fully configurable during MBE by altering deposition factors such as: dot size, density, material/s, multi-layer periodicity, and density-ofstates. ${ }^{9}$ QD's have been shown to exhibit ultrafast recovery times, ${ }^{10-12}$ and InAs QD layers integrated into bulk GaAs can act analogously to mid-gap carrier trapping sites. This allows a severe reduction in charge carrier lifetimes with a comparatively small reduction in average carrier mobility ${ }^{13}$ as the vast majority of the bulk lattice volume may be of high crystalline quality, unlike that of LT-GaAs. For example, self-assembled nanoislands of ErAs acting as lattice defects in bulk GaAs can limit carrier lifetimes down to around 0.15 ps with a layer period reduced to $20 \mathrm{~nm} .{ }^{14}$ Furthermore, ErAs/GaAs grown in this way allows sub-picosecond modulation for use in $\mathrm{THz}$ photomixer devices. ${ }^{15}$ Controlling the integration of carrier trapping sites, which are implanted discretely and independently from the PC medium during MBE allows another dimension of freedom regarding the appropriation of, for example: PC medium; carrier lifetimes; quantum gain; as well as thermal and structural parameters (e.g., resonant cavity designs). We consider here a preliminary alternating-layer photomixer test design based on GaAs and $\mathrm{In}_{0.15} \mathrm{Ga}_{0.85} \mathrm{As}$ as the PC medium and InAs QD's as the lattice defect/trapping sites.

The photomixer was pumped by two AlGaAs distributed feedback (DFB) laser diodes (LD's) ${ }^{16}$ at around $847 \mathrm{~nm}$ and $850 \mathrm{~nm}$ longitudinal wavelengths, which were electrically driven at a current of $80 \mathrm{~mA}$, focused and coupled using AntiReflective coated aspheric lenses then spatially combined in a 2:1 fiber coupler. LD wavelengths could each be tuned within a range of around $0.66 \mathrm{~nm}$ by altering the operating temperature using a low-noise thermoelectric cooler between $17^{\circ} \mathrm{C}$ and $25^{\circ} \mathrm{C}$. This allowed the $\mathrm{THz}$ difference frequency to be tuned between $0.76 \mathrm{THz}$ and $1.31 \mathrm{THz}$. Each laser linewidth was less than $0.2 \mathrm{~nm}$. At $25^{\circ} \mathrm{C}$, each laser output up to $100 \mathrm{~mW}$ of optical power. DFB lasers have been used in this way previously to demonstrate telecoms-wavelength tunable heterodyning up to 


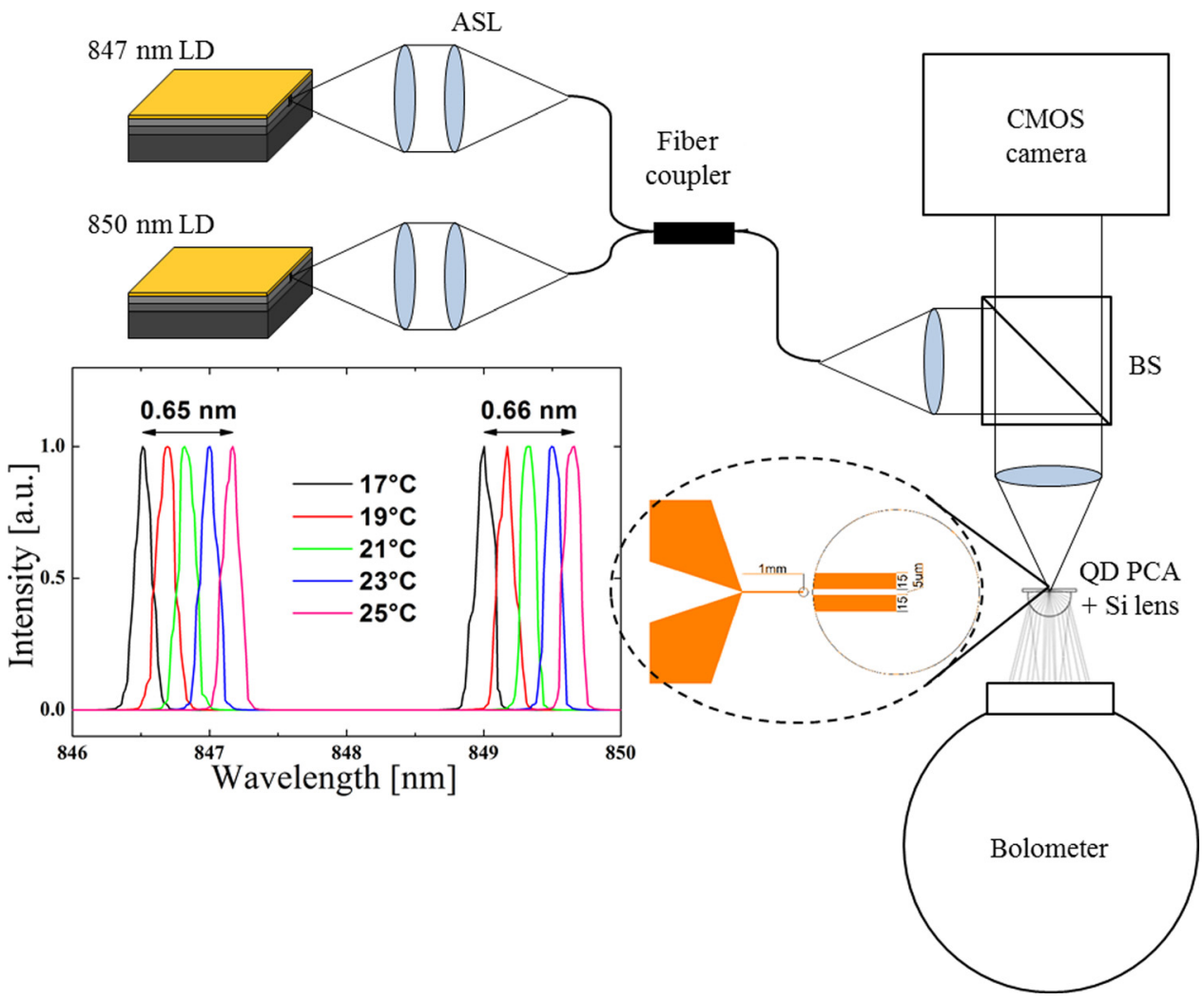

FIG. 1. Experimental setup and (inset) LD pump wavelength ranges with $\mathrm{LD}$ temperature variation. ASL-aspheric lenses; BS-beamsplitter cube; and PCA—photoconductive antenna.
$0.9 \mathrm{THz}$ in a waveguide-integrated photodiode antenna ${ }^{17}$ as the narrow linewidths associated with DFB structures allow narrowband $\mathrm{THz}$ signals to be generated, which is advantageous in a tunable CW THz source (Fig. 1 inset).

The test photomixer structure was composed of: a thin top layer of $30 \mathrm{~nm} \mathrm{LT-GaAs} \mathrm{to} \mathrm{ensure} \mathrm{a} \mathrm{high} \mathrm{breakdown}$ field at the surface; 40 periods of InAs QD's with adjacent $5 \mathrm{~nm}$ thickness $\mathrm{In}_{0.15} \mathrm{Ga}_{0.85} \mathrm{As}$ PC wetting layers and $35 \mathrm{~nm}$ GaAs capping/separating layers; a $35 \mathrm{~nm}$ GaAs spacer layer; a 25-period AlAs/GaAs distributed Bragg reflector (DBR) to retroreflect any optical pump not absorbed by the active PC volume; and the semi-insulating GaAs substrate. PCA Au:Ti electrodes were defined over the top surface using standard UV lithography, a wet-etching processes and a post-process anneal at $410{ }^{\circ} \mathrm{C}$ for $3.5 \mathrm{~min}$ to ensure Ohmic contact with the photomixer surface. The electrode geometries used in the preliminary studies were $1 \mathrm{~mm}$-long coplanar striplines (CPS) with $15 \mu \mathrm{m}$ electrode width and a $5 \mu \mathrm{m}$ PC gap. An $8 \mathrm{~mm}$-diameter hyperhemispherical silicon lens was abutted to the "back" of Semi-Insulating GaAs wafer face to enhance collection and outcoupling of the generated $\mathrm{THz}$ signal.

The polarisation and coupled power from each LD was monitored and kept equal, and the combined beam was focused onto the active gating area of the PCA. This was further optimised by monitoring the reduction in PCA resistance from above $10 \mathrm{M} \Omega$ to a few hundred $\mathrm{k} \Omega$ as the pump alignment and power was increased. A square-wave bias with a frequency of $197 \mathrm{~Hz}$ was applied across the electrodes and simultaneously used as a trigger for a lock-in amplifier (LIA). The $\mathrm{THz}$ signal was detected using a liquid heliumcooled germanium bolometer, the response from which was electrically chopped by the PCA bias signal and monitored using the LIA. The entrance window of the bolometer is fitted with a mesh bandpass filter with frequency range 0.6-10 THz to transmit. Photomixer device theory gives us a prediction of the generated $\mathrm{THz}$ power trend as these factors are altered, which is an increasing $\mathrm{THz}$ output power with increasing PCA bias and increasing optical pump power. (a)

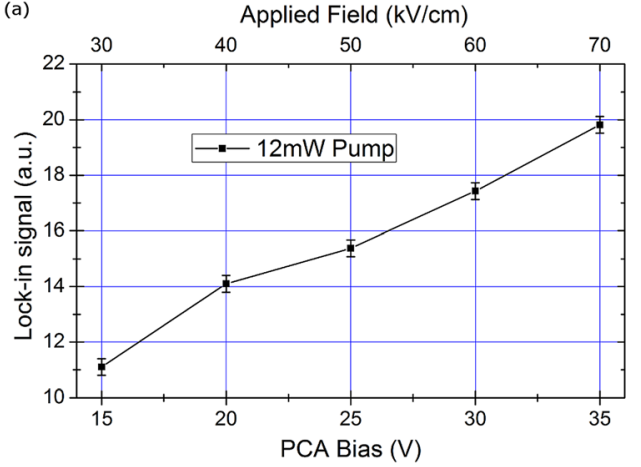

(b)

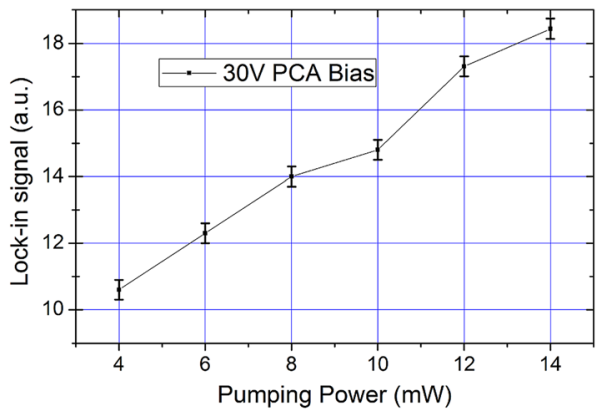

FIG. 2. Typical CW THz output power trends from a QD photomixer test device. (a) Monitored as the optical pump power is held constant and the PCA bias and accelerating E-field is increased; (b) as the PCA bias is held constant and the optical pump power is increased. 


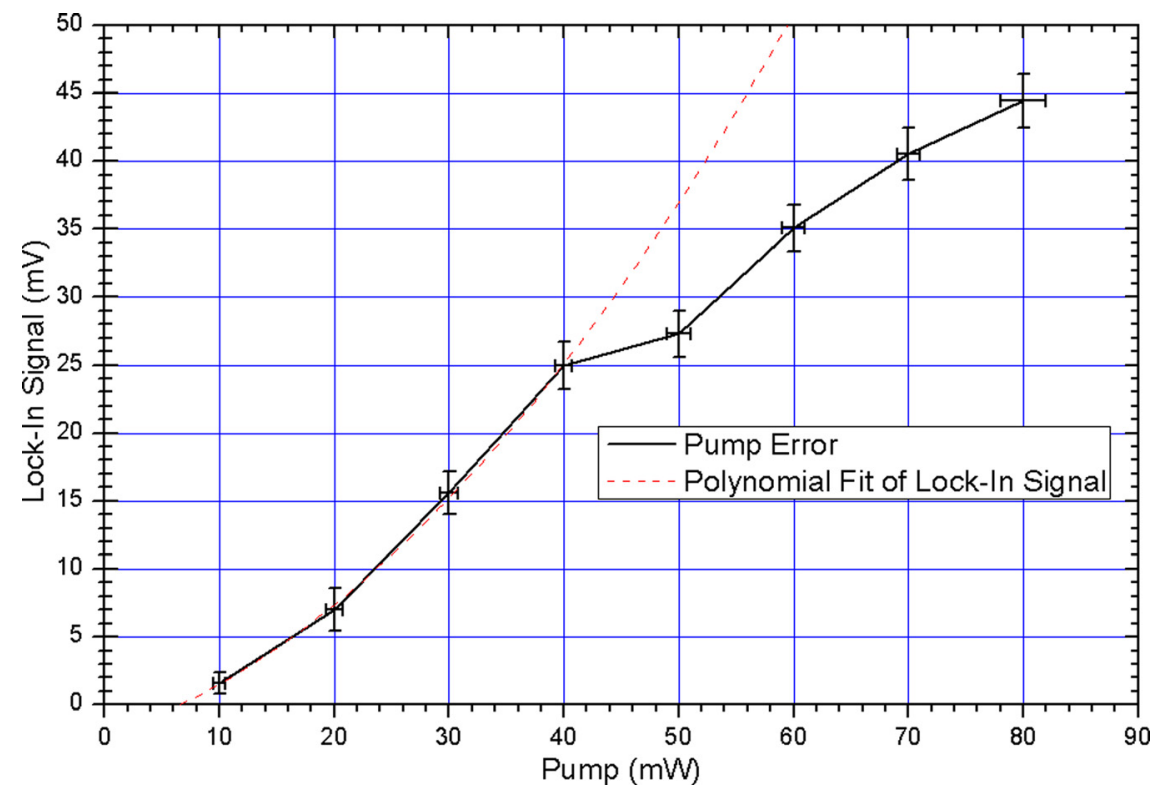

FIG. 3. Example THz output power trend observed as the structure is optically pumped by a Ti:Sapphire laser, operating at $780 \mathrm{~nm}$ with a pulsewidth of $150 \mathrm{fs}$. Initially, the square-law dependence of output power on optical pump power is observed, then the structure begins to exhibit $\mathrm{THz}$ output saturation characteristics.
Typical $\mathrm{THz}$ output power trends are exemplified in Figure 2. It was found that the response from the bolometer increased predictably and repeatably as the PCA bias was increased from $15 \mathrm{~V}$ to $35 \mathrm{~V}$, and likewise as the optical pump power was increased from $4 \mathrm{~mW}$ to $14 \mathrm{~mW}$. The results displayed here correspond to a peak optical pump difference frequency of $\sim 1.04 \mathrm{THz}$, which is well within the frequency limitations imposed by the expected carrier lifetime of $\sim 0.425 \mathrm{ps}(\tau \approx 2.35 \mathrm{THz})$.

For further clarification of the ultrafast behaviour of the structure, the antenna was driven using a mode-locked Ti:Sapphire laser operating at $780 \mathrm{~nm}$ wavelength, with pulses of 150 fs duration at a repetition rate of $96 \mathrm{MHz}$. This pump beam was focused as before and the antenna bias and optical pump power were altered in the same way as the CW setup, and the $\mathrm{THz}$ output signal was monitored using the bolometer and LIA. An initial square-law dependence of the output signal on optical power and antenna bias was observed, after which (above $40 \mathrm{~mW}$ pump power) the structure exhibited some output saturation behaviour, exemplified in Figure 3, which is typically to be expected in an operational PC $\mathrm{THz}$ antenna. The effective PC material in this case is the high-temperature-grown GaAs between the QD layers, which on its own would not operate as an ultrafast PC switch due to the comparatively long carrier lifetimes. It is, therefore, likely that the inclusion of QD's in this case has allowed the ultrafast carrier capture mechanism ${ }^{10}$ by which $\mathrm{THz}$ signals may be generated in the antenna. It follows then that it is possible one may utilise the InAs QD's themselves also as the PC medium. The photoluminescence (PL) spectrum for this structure is shown in Figure 4, which indicates it could potentially allow for ultrafast photomixing processes to be generated using longer pump wavelengths approaching $1.3 \mu \mathrm{m}$. Interestingly, coherent $\mathrm{THz}$ emission spectroscopy has observed $\mathrm{THz}$ signals from the structure as it is pumped by $150 \mathrm{fs}$ pulses over a wavelength range of $650 \mathrm{~nm}$ to $1180 \mathrm{~nm}$, and in addition to the detected THz output signal as the structure is pumped up to around $880 \mathrm{~nm}$, the upper limit of the GaAs absorption, distinct $\mathrm{THz}$ emission peaks were observed as the pump wavelength approaches the excited and second-excited state peak PL wavelengths of the InAs QD's. ${ }^{18}$

These results demonstrate the potential for increased optical-to-THz efficiency offered by configurable QD-based photomixer structures over existing materials. The main advantages of these structures include the configurability of the PC region

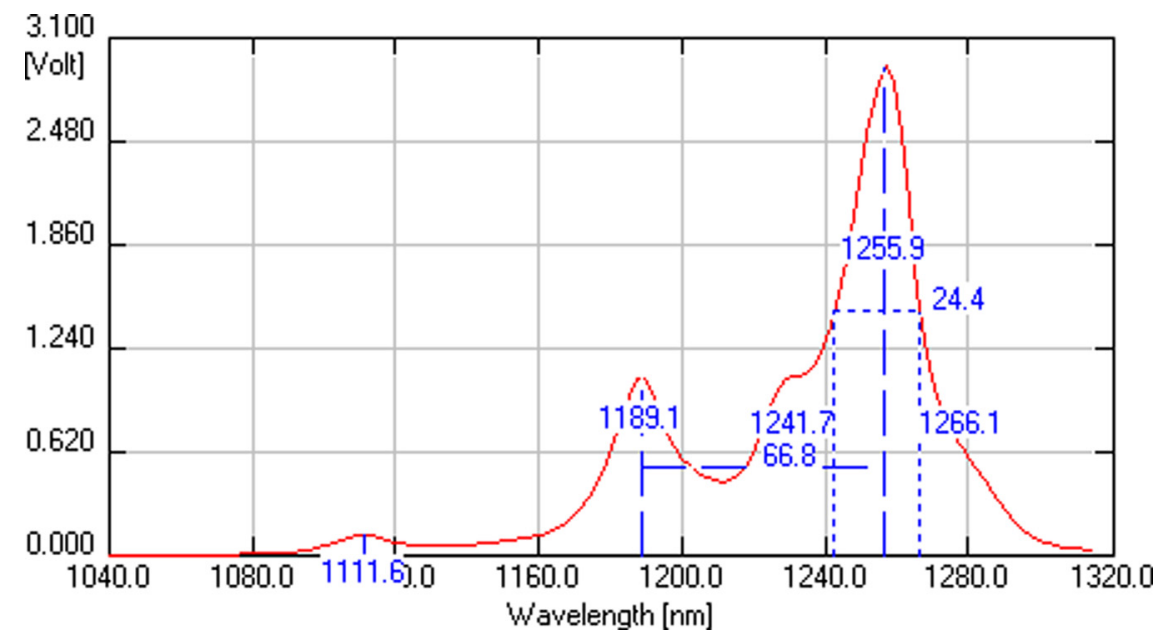

FIG. 4. PL spectrum from the QD-based structure showing the distinct ground state, excited state, and second-excited state PL peaks at around $1256 \mathrm{~nm}$, $1189 \mathrm{~nm}$, and $1111 \mathrm{~nm}$, respectively. 
to alter peak absorption wavelengths as well as photocarrier lifetimes and quantum gain by altering QD layer periodicity. Separate configuration of the PC region could allow the use of longer-wavelength QD-based pump sources, which are broadly tunable in their mean longitudinal wavelengths as well as difference frequencies. ${ }^{5}$ This opens up the potential for highly tunable, ultra-compact, fully integrated, or on-chip $\mathrm{CW} \mathrm{THz}$ transceivers similar to that presented, for example, in Ref. 17 as both the QD-based LD's and photomixers operate around the same optical emission and pump wavelengths.

In conclusion, generation of continuous wave radiation was demonstrated, at a frequency of $1.04 \mathrm{THz}$ from a semiconductor optical heterodyne device, which comprises 40 periods of InAs quantum dot layers separated by $40 \mathrm{~nm}$, with PC InGaAs wetting layers between GaAs capping layers. The photomixer was optically pumped by two spatially combined AlGaAs DFB LD's at around $847 \mathrm{~nm}$ and $850 \mathrm{~nm}$ longitudinal wavelengths.

This project was funded by EPSRC under Grant EP/ H015795/1. The authors would like to thank Dr D. Livshits of Innolume $\mathrm{GmbH}$, Germany, for production of the QD photomixer structure. We would also like to thank: $\mathbf{M}^{2}$ Lasers, UK, for their part-funding support and provision of equipment; and Compugraphics, UK, for production of fine-feature photomasks, which made the production of sample antennas possible.

${ }^{1}$ E. R. Brown, F. W. Smith, and K. A. McIntosh, J. Appl. Phys. 73(3), 1480 (1993).

${ }^{2}$ R. Leyman, N. Bazieva, T. Kruczek, G. S. Sokolovskii, and E. U. Rafailov, Recent Pat. Signal Process. 2(1), 12 (2012).
${ }^{3}$ D. H. Auston, Appl. Phys. Lett. 26(3), 101 (1975).

${ }^{4}$ L. Rui, C. M. Gu, L. R. He, W. Sen, W. Z. Shen, O. Hiroshi, and Q. X. Guo, Acta Phys. Sin. 53 (4), 1217 (2004).

${ }^{5}$ R. Leyman, D. I. Nikitichev, N. Bazieva, and E. U. Rafailov, Appl. Phys. Lett. 99(17), 171107 (2011).

${ }^{6}$ P. R. Smith, D. H. Auston, and M. C. Nuss, IEEE J. Quantum Electron. 24(2), 255 (1988).

${ }^{7}$ F. W. Smith, A. R. Calawa, C. L. Chen, M. J. Manfra, and L. J. Mahoney, IEEE Electron Device Lett. 9(2), 77 (1988).

${ }^{8}$ F. W. Smith, H. Q. Le, V. Diadiuk, M. A. Hollis, A. R. Calawa, S. Gupta, M. Frankel, D. R. Dykaar, G. A. Mourou, and T. Y. Hsiang, Appl. Phys. Lett. 54(10), 890 (1989).

${ }^{9}$ A. R. Kovsh, N. N. Ledentsov, S. S. Mikhrin, A. E. Zhukov, D. A. Livshits, N. A. Maleev, M. V. Maximov, V. M. Ustinov, A. E. Gubenko, I. M. Gadjiev, E. L. Portnoi, J. S. Wang, J. Chi, D. Ouyang, D. Bimberg, and J. A. Lott, in Physics and Simulation of Optoelectronic Devices XII, edited by M. Osinski, H. Amano, and F. Henneberger (Spie-Int Soc Optical Engineering, Bellingham, 2004), Vol. 5349, pp. 31.

${ }^{10}$ E. U. Rafailov, S. J. White, A. A. Lagatsky, A. Miller, W. Sibbett, D. A. Livshits, A. E. Zhukov, and V. M. Ustinov, IEEE Photon. Technol. Lett. 16(11), 2439 (2004).

${ }^{11}$ H. P. Porte, P. Uhd Jepsen, N. Daghestani, E. U. Rafailov, and D. Turchinovich, Appl. Phys. Lett. 94(26), 262104 (2009).

${ }^{12}$ M. C. Hoffmann, B. S. Monozon, D. Livshits, E. U. Rafailov, and D. Turchinovich, Appl. Phys. Lett. 97(23), 231108 (2010).

${ }^{13}$ D. Turchinovich, K. Pierz, and P. Uhd Jepsen, Phys. Status Solidi C 0(5), 1556 (2003).

${ }^{14}$ C. Kadow, S. B. Fleischer, J. P. Ibbetson, J. E. Bowers, A. C. Gossard, J. W. Dong, and C. J. Palmstrom, Appl. Phys. Lett. 75(22), 3548 (1999).

${ }^{15}$ C. Kadow, A. W. Jackson, A. C. Gossard, J. E. Bowers, S. Matsuura, and G. A. Blake, Physica E 7(1-2), 97 (2000).

${ }^{16}$ A. Klehr, J. Fricke, A. Knauer, G. Erbert, M. Walther, R. Wilk, M. Mikulics, and M. Koch, IEEE J. Sel. Top. Quantum Electron. 14(2), 289 (2008).

${ }^{17}$ D. Stanze, A. Deninger, A. Roggenbuck, S. Schindler, M. Schlak, and B. Sartorius, J. Infrared Millim. Terahertz Waves 32(2), 225 (2011).

${ }^{18}$ G. Molis, A. Arlauskas, A. Krotkus, R. Leyman, N. Bazieva, and E. U. Rafailov, in 37th International Conference on Infrared, Millimeter, and Terahertz Waves, 2012. IRMMW-THz 2012 (Wollongong, Australia, 2012). 